\title{
On the cardinality of sumsets in torsion-free groups
}

\author{
Károly J. Böröczky*, Péter P. Pálfy† Oriol Serra
}

\begin{abstract}
Let $A, B$ be finite subsets of a torsion-free group $G$. We prove that for every positive integer $k$ there is a $c(k)$ such that if $|B| \geq c(k)$ then the inequality $|A B| \geq|A|+|B|+k$ holds unless a left translate of $A$ is contained in a cyclic subgroup. We obtain $c(k)<c_{0} k^{6}$ for arbitrary torsion-free groups, and $c(k)<c_{0} k^{3}$ for groups with the unique product property, where $c_{0}$ is an absolute constant. We give examples to show that $c(k)$ is at least quadratic in $k$.
\end{abstract}

\section{Introduction}

Let $G$ be a torsion-free group written multiplicatively, and let $|\cdot|$ denote the cardinality of a finite set. A basic problem in Additive Combinatorics is to estimate the cardinality of $A B=\{a b: a \in A, b \in B\}$ of two finite sets $A, B \subset G$ in terms of $|A|$ and $|B|$. A basic notion is a progression with ratio $r \neq 1$ and length $n$, which is a set of the form $\left\{a, a r, \ldots, a r^{n-1}\right\}$ where $a$ and $r$ commute.

Let us review some related results if $G$ is abelian. In this case we have the simple inequality

$$
|A B| \geq|A|+|B|-1,
$$

with equality if and only if $A$ and $B$ are progressions with common ratio. Following Ruzsa [21, we call the minimal rank of a subgroup whose some coset contains $A$ the dimension of $A$. According to Freiman [5], if the dimension of $A$ is $d$, then

$$
\left|A^{2}\right| \geq(d+1)|A|-\left(\begin{array}{c}
d+1 \\
2
\end{array}\right) .
$$

${ }^{*}$ Supported by OTKA grants 068398 and 75016, and by the EU Marie Curie FP7 IEF grant GEOSUMSETS

${ }^{\dagger}$ Supported by OTKA grant NK72523 
This estimate is optimal. It follows that if $\left|A^{2}\right| \leq 3|A|-4$, then $A$ is contained in some coset of a cyclic group. Actually, even a progression of length $2|A|-3$ contains $A$ according to the $(3 k-4)$-theorem of Freiman [5]. More precise structural information on $A$ is available if $\left|A^{2}\right|=2|A|+n$ for $0 \leq n \leq|A|-4$ by Freiman [7], for example, $A$ is contained in a progression of length $|A|+n+1$.

The inequality (11) was generalized to a pair of sets by Ruzsa [20] who proved that if $|A| \geq|B|$, and the dimension of $A B$ is $d$, then

$$
|A B| \geq|A|+d|B|-\left(\begin{array}{c}
d+1 \\
2
\end{array}\right) .
$$

By requiring additionally that the smaller set $B$ is $d$-dimensional, Gardner and Gronchi [8] proved a discrete version of the Brunn-Minkowski inequality which shows that

$$
|A B| \geq|A|+(d-1)|B|+(|A|-d)^{(d-1) / d}(|B|-d)^{1 / d}-\left(\begin{array}{l}
d \\
2
\end{array}\right) .
$$

Additional lower bounds with stronger geometric requirements on the sets $A$ and $B$ have been also obtained by Matolcsi and Ruzsa [16] and Green and Tao [9].

In the non-abelian case the situation is much less understood. Kempermann [15] implies in the case of any torsion-free group $G$ that

$$
|A B| \geq|A|+|B|-1 \text {. }
$$

Brailovsky and Freiman [1] characterized the extremal sets in the inequality (41) by showing that, if $\min \{|A|,|B|\} \geq 2$ then, up to appropriate left and right translations, both $A$ and $B$ are progressions with common ratio. In particular, $A$ and $B$ lie in a left and a right coset, respectively, of a cyclic subgroup.

The analogy with the abelian case was extended in Hamidoune, Lladó and Serra [14] to the inequality

$$
|A B| \geq|A|+|B|+1
$$

if $|B| \geq 4$, and $A$ is not contained in some left coset of a cyclic subgroup.

These known facts are connected with the following conjecture of Freiman (personal communication), extending the $(3 k-4)$-theorem above. 
Conjecture 1 Let $A$ be a finite subset of a torsion-free group with $|A| \geq 4$. If

$$
\left|A^{2}\right| \leq 3|A|-4
$$

then $A$ is covered by a progression of length at most $2|A|-3$.

By using the so-called isoperimetric method, see Hamidoune [11, [12] or [13, we obtain the following results:

Theorem 2 For any integer $k \geq 1$ there exists a $c(k)$ such that the following holds. If $G$ is a torsio-free group, $A \subset G$ is not contained in a left coset of any cyclic subgroup, $B \subset G$ has more than $c(k)$ elements, then

$$
|A B|>|A|+|B|+k .
$$

Remark Our current methods yield $c(k) \leq 32(k+3)^{6}$.

Note that, in Theorem 2, the assumption on $A$ not being contained in a left coset of a cyclic group is crucial. For example, if $A$ is a progression of length at most $k+2$ with ratio $r \neq 1$, and $B$ is the union of two $r$-progressions of arbitrary length, then $|A B| \leq|A|+|B|+k$.

The value of the lower bound $c(k)$ can be improved for unique product groups. Recall that a group $G$ has the unique product property if, for every pair of finite sets $A, B \subset G$, there is an element $g \in A B$ which can be uniquely expressed as a product of an element of $A$ and an element of $B$. In this case, $G$ is torsion-free. We note that every right linearly orderable group has the unique product property, and any residually finite word hyperbolic group has a finite index unique product subgroup, according to $\mathrm{T}$. Delzant [4]. On the other hand, it was first shown by Rips and Segev [18] that not all torsion-free groups have the unique product property, and S.D. Promislow [17] even provided an explicit construction for such an example. In addition, unique product groups are discussed in A. Strojnowski [22, S.M. Hair [10], and W. Carter [3]. For unique product groups, the bound on $c(k)$ in Theorem 2 can be reduced to a cubic polynomial on $k$; namely, Lemma 12 yields that

$$
c(k) \leq 4(2 k+3)^{3} \text { if } G \text { is a unique product group. }
$$

We note that it can be deduced with the help of (3) that in abelian torsionfree groups, the optimal order of $c(k)$ is quadratic. The $c(k)$ in Theorem 2 
is at least of quadratic order also for non-abelian unique product groups, as the following example shows.

We consider the Klein bottle group $G_{0}=\left\langle u, v \mid u^{-1} v u=v^{-1}\right\rangle$, and hence $v u=u v^{-1}$ and $v^{-1} u=u v$. Since $\langle v\rangle$ is a normal subgroup with factor isomorphic to $\mathbb{Z}, G_{0}$ is a non-abelian unique product group. Let $A=\{1, u, v\}$ and $B=\left\{u^{i} v^{j}: i, j=0,1, \ldots, m-1\right\}$ for $m \geq 1$. Then $|B|=m^{2}$ and $A B=\cup_{i, j=0,1, \ldots, m-1}\left\{u^{i} v^{j}, u^{i+1} v^{j}, u^{i} v^{j+(-1)^{i}}\right\}$, thus

$$
|A B|=m^{2}+2 m=|A|+|B|+2|B|^{\frac{1}{2}}-3 .
$$

In line with Conjecture 1, we conjecture that, if $A=B$, then the lower bound on $|B|$ in Theorem 2 can be replaced by a bound linear in $k$. We construct an example in the group $G_{0}$ above to indicate, what to expect in Theorem 2 in this case.

For $m \geq 1$, let $A=P \cup v u Q$ where $P=\left\{u^{i}: i=0,1, \ldots, 2 m\right\}$ and $Q=$ $\left\{u^{2 i}: i=0,1, \ldots, m-1\right\}$, and hence $|A|=3 m+1$. Since $v$ commutes with $u^{2}$, we have $(v u Q)(v u Q)=(v u v) u Q^{2}=u^{2} Q^{2} \subset P^{2}$. Moreover, denoting by $P_{0}$ and $P_{1}$ the set of even and odd powers of $u$ in $P$, respectively, we have $P v u Q=v u P_{0} Q \cup v^{-1} u P_{1} Q \subset v u Q P \cup v^{-1} u P_{1} Q$. It follows that

$$
\left|A^{2}\right|=\left|P^{2}\right|+|Q P|+\left|P_{1} Q\right|=10 m-1=\frac{10}{3}|A|-\frac{13}{3} .
$$

We note that the above example seems to match a conjecture of Freiman 6], which would yield that $A$ is the union of two progressions provided that $\left|A^{2}\right|<\frac{10}{3}|A|-5$.

In the direction of Conjecture 1 for a torsion-free group $G$, our results yield the following.

Corollary 3 If $A$ is a subset of a torsion-free group with $|A| \geq 6^{6}$, and $\left|A^{2}\right|=2|A|+n$ for $0 \leq n \leq 2^{-5 / 6}|A|^{1 / 6}-3$, then $A$ is contained in a progression of length $|A|+n+1$.

Remark In unique product groups, the conditions are $|A| \geq 6^{3}$ and $0 \leq n \leq 2^{-5 / 3}|A|^{1 / 3}-\frac{3}{2}$.

If $A$ is a finite subset of a torsion-free group $G$, then Corollary 3 provides strong structural information when $\left|A^{2}\right|$ is very close to $2|A|$. This has been made possible in part by the known structural properties in abelian groups. 
Now if $G$ is abelian and $\left|A^{2}\right|<K|A|$ for some $K>3$ then still strong structural properties have been established by Freiman [5] using multidimensional progressions, see the monograph of Tao and $\mathrm{Vu}$ [24] or the survey by Ruzsa [21] for recent developments. But if $G$ is any torsion-free group and $K \geq \frac{10}{3}$, then $A$ may not be contained in an abelian subgroup. Actually, it is still not completely understood, what to expect, in spite the results about some specific groups (see Breuillard and Green [2], or Tao's blog [23]).

\section{Atoms and fragments}

For this section, we fix a torsion-free group $G$.

For $n \geq 1$ and a finite non-empty set $C \subset G$, the $n$-th isoperimetric number of $C$ is defined to be

$$
\kappa_{n}(C)=\min \{|X C|-|X|: X \subset G \text { and }|X| \geq n\} .
$$

A finite set $V \subset G$ is an $n$-fragment for $C$, if $|V| \geq n$ and $|V C|-|V|=\kappa_{n}(C)$. In addition an $n$-fragment of minimal cardinality is an $n$-atom for $C$.

Naturally, if $U$ is an $n$-atom for $C$, then $x U$ is also an $n$-atom for $C y$ for any $x, y \in G$. In what follows, we present simple statements about atoms. For the sake of completeness, we verify even the known ones, except for the following crucial property of atoms, due to Hamidoune [11]: If $U$ is an $n$-atom and $F$ is an $n$-fragment for a finite nonempty subset $C \subset G$, then

$$
\text { either } U \subset F \text { or }|U \cap F| \leq n-1 \text {. }
$$

This property has the following useful consequence.

Corollary 4 For a torsion-free group $G$ and $n \geq 1$, if $U$ is an $n$-atom for $C \subset G$ and $g \in G \backslash 1$, then $|U \cap g U| \leq n-1$.

For right translations we have a weaker result.

Lemma 5 For a torsion-free group $G$ and $n \geq 2$, if $U$ is an n-atom for $C \subset G$ and $g \in G \backslash 1$, then

$$
|U \cap U g| \leq \frac{n-2}{n-1}|U|+\frac{1}{n-1} \leq \frac{n-1}{n}|U| .
$$


Remark In particular, if $n=2$, then $|U \cap U g| \leq 1$.

Proof: Let us partition $U$ into the maximal left $g$-progressions $U_{1}, \ldots, U_{m}$, where $U_{i}=\left\{h_{i}, h_{i} g, \ldots, h_{i} g^{\alpha_{i}}\right\}, i=1, \ldots, m$. In particular, $U_{i} \cap U_{j} g=\emptyset$ for $i \neq j$. We may assume that $\left|U_{1}\right| \geq\left|U_{i}\right|, i=2, \ldots, m$ and that $h_{1}=1$.

It follows by Corollary 4 that

$$
\left|U_{1} \cap g U_{1}\right| \leq n-1,
$$

thus $\left|U_{1}\right| \leq n$. In addition, for $i \geq 2$, we have

$$
\left|U_{1} \cap h_{i}^{-1} U_{i}\right| \leq n-1,
$$

thus $\left|U_{i}\right| \leq n-1$. Therefore $|U| \leq m(n-1)+1$ and

$$
|U \cap U g|=|U|-m \leq \frac{n-2}{n-1}|U|+\frac{1}{n-1} \leq \frac{n-1}{n}|U|,
$$

as claimed. Q.E.D.

The minimality of the cardinality of atoms directly yields (see [11] or [14])

Lemma 6 If $U$ is an $n$-atom for $C \subset G, n \geq 1$, in a torsion-free group $G$, and $|U|>n$, then any element in $U C$ can be represented in at least two ways as a product of an element of $U$ and an element of $C$.

We deduce two rough, but useful estimates about 2-atoms which can be found in [1] as well.

Lemma 7 If $U$ is a 2-atom for $C \subset G,|C| \geq 3$, in a torsion-free group $G$, then $|U| \leq|C|-1$.

Proof: We may assume that $|U|>2$ and $1 \in C$, and hence $U \subset U C$. According to Lemma 6, for any $u \in U$, there are $v_{u} \in U$ and $c_{u} \in C \backslash 1$ such that $u=v_{u} c_{u}$. If $c_{u}=c_{w}$ for $u \neq w \in U$, then $\left\{v_{w}, w\right\} \subset U \cap v_{w} v_{u}^{-1} U$, contradicting Corollary 4 . Therefore $u \mapsto c_{u}$ is an injective map from $U$ into $C \backslash 1$. Q.E.D.

For any non-empty $C \subset G$, let $C^{-1}=\left\{g^{-1}: g \in C\right\}$.

Lemma 8 If $U$ is a 2-atom for $C \subset G,|C| \geq 3$, in a torsion-free group $G$, and $|U C| \leq|U|+|C|+k$, then $|U| \leq k+3$. 
Proof: Let $V$ be a 2-atom for $U^{-1}$ with $1 \in V$, thus $\left|V U^{-1}\right|-|V|-\left|U^{-1}\right| \leq\left|C^{-1} U^{-1}\right|-\left|C^{-1}\right|-\left|U^{-1}\right|=|U C|-|U|-|C| \leq k$.

If $V=\{1, g\}$ with $g \neq 1$, then Lemma 5 yields

$$
2|U|-1 \leq\left|U V^{-1}\right|=\left|V U^{-1}\right| \leq k+2+|U|,
$$

which in turn implies $|U| \leq k+3$. If $|V| \geq 3$, then Lemma 5 and Lemma 7 yield

$|U|+(|U|-1)+(|U|-2) \leq\left|U V^{-1}\right|=\left|V U^{-1}\right| \leq k+|V|+|U| \leq k+2|U|-1$,

which in turn implies $|U| \leq k+2$. Q.E.D.

Now we extend Lemma 8 to $n$-atoms, which extension is the only novel result of this section.

Proposition 9 If $U$ is an n-atom for $C \subset G,|C| \geq 3$ and $n \geq 3$, in a torsion-free group $G$, and $|U C| \leq|U|+|C|+k$, then $|U| \leq n(2 k+3)$.

Proof: Let $V$ be a 2-atom for $U^{-1}$, hence

$$
\left|V U^{-1}\right|-|V|-\left|U^{-1}\right| \leq\left|C^{-1} U^{-1}\right|-\left|C^{-1}\right|-\left|U^{-1}\right| \leq k .
$$

It follows by Lemma 8 that $|V| \leq k+3$. Moreover, by Lemma 5 , we have $\left|U V^{-1}\right| \geq 2|U|-\frac{n-1}{n}|U|$. Hence,

$$
\frac{n+1}{n}|U| \leq\left|U V^{-1}\right|=\left|V U^{-1}\right| \leq|U|+|V|+k \leq|U|+2 k+3,
$$

thus $|U| \leq n(2 k+3)$. Q.E.D.

All these statements about atoms would readily follow from the following conjecture of Y.O. Hamidoune [13].

Conjecture 10 Any n-atom in a torsion-free group has cardinality $n$.

We recall that a group $G$ has the unique product property if for any finite non-empty sets $A, B \subset G$, there is a $g \in A B$ that can be represented in a unique way in the form $a b$ with $a \in A$ and $b \in B$. In this case $G$ is torsion-free. It follows by Lemma 6 that unique product groups satisfy Conjecture 10. 


\section{Small product sets}

The proof of Theorem 2 together with an estimate of $c(k)$ will follow from the following Lemma and the estimations on the size of atoms in the previous section.

Lemma 11 Let $G$ be a torsion-free group. Suppose that $A \subset G$ with $|A|=3$ is not contained in a left coset of any cyclic subgroup of $G$. For $d \geq 3$ and any finite set $B \subset G$ of cardinality greater than $4 d^{3}$, we have

$$
|A B|>|B|+d \text {. }
$$

Proof: We suppose that $|A B| \leq|B|+d$, and seek a contradiction. We may assume that $A=\{1, u, v\}$, where $\langle u, v\rangle$ is not cyclic.

For $g \in G$, we write $B_{g}=B \backslash g^{-1} B=\{x \in B \mid g x \notin B\}$. Since $|B \cup u B| \leq$ $|B|+d$, we see that $\left|B_{u}\right| \leq d$. Similarly, $\left|B_{u^{-1}}\right| \leq d$, as $\left|u^{-1} B \cup B\right|=$ $|B \cup u B| \leq|B|+d$, and, of course, $\left|B_{v}\right|,\left|B_{v^{-1}}\right| \leq d$ also hold. Since $B$ is finite, for any $x \in B$ the coset $\langle u\rangle x$ must contain an element of $B_{u}$, hence the elements of $B$ belong to at most $d$ cosets of $\langle u\rangle$, and similarly for $\langle v\rangle$. Therefore there exists an $x_{0} \in B$ such that

$$
\left|B \cap\langle u\rangle x_{0} \cap\langle v\rangle x_{0}\right| \geq|B| / d^{2}>4 d .
$$

In order to simplify notation, by replacing $B$ with $B x_{0}^{-1}$, we may assume without loss of generality that $x_{0}=1$. Let $Z=\langle u\rangle \cap\langle v\rangle$, and $B_{0}=B \cap Z$. We have $\left|B_{0}\right|>4 d$. Elements of $Z$ are powers of both $u$ and $v$, hence $Z$ is contained in the center of $H=\langle u, v\rangle$. As $Z \neq\{1\}$ and $A$ does not generate a cyclic group, we deduce that $u$ and $v$ do not commute.

We are going to show that $B Z \supseteq H$. Take an element $g \in H$, and let us choose a word of shortest length $a_{n} a_{n-1} \cdots a_{2} a_{1}$, where each $a_{i}$ is one of $u, u^{-1}, v, v^{-1}$, in the coset $g Z$. Then the cosets $Z, a_{1} Z, a_{2} a_{1} Z, \ldots$, $a_{n} a_{n-1} \cdots a_{1} Z$ are pairwise disjoint. To any $x \in B_{0}$, we assign the sequence $S_{x}=\left\{a_{i} a_{i-1} \cdots a_{1} x\right\}_{i=0,1, \ldots, n}$, which sequences are pairwise disjoint as $x$ runs through $B_{0}$. If $a_{n} a_{n-1} \cdots a_{1} x \notin B$, then there is a smallest $i \in\{1, \ldots, n\}$ such that $a_{i} \cdots a_{1} x \notin B$. It follows that $S_{x}$ has an element in $B_{a_{i}} \subset B_{u} \cup B_{u^{-1}} \cup B_{v} \cup B_{v^{-1}}$, namely, $x$ if $i=1$, and $a_{i-1} \cdots a_{1} x$ if $i \geq 2$. Since $\left|B_{0}\right|>4 d \geq\left|B_{u}\right|+\left|B_{u^{-1}}\right|+\left|B_{v}\right|+\left|B_{v^{-1}}\right|$, and $S_{x} \cap S_{y}=\emptyset$ for $x \neq y$ in $B_{0}$, there exists an $x \in B_{0}$ such that $a_{n} a_{n-1} \cdots a_{1} x \in B$. We conclude that $g \in a_{n} a_{n-1} \cdots a_{1} x Z \subset B Z$. 
Now the index of the central subgroup $Z$ in $H$ is finite (bounded by $|B|$ ), so the center has finite index in $H$. According to a classical theorem of Schur (see, e.g., Robinson [19, Theorem 10.1.4]), this implies that the commutator subgroup of $H$ is finite. If the commutator subgroup is 1, then $H$ is abelian, and if the commutator subgroup is non-trivial, then we have some torsion elements. In any case, we have contradicted the assumptions on $A$ and $G$, and hence proved the lemma. Q.E.D.

Proof of Theorem 2: Without loss of generality we may assume that $1 \in A$. Then $\langle A\rangle$ is not cyclic by our assumption. Let $B \subset G$ be a finite set with $|B|>32(k+3)^{6}$. If $B$ is contained in some right coset of a cyclic subgroup $H$, then $A$ intersects at least two left cosets of $H$. Let $A_{1}$ be one of these intersections. Then using (4) we get

$$
|A B|=\left|A_{1} B\right|+\left|\left(A \backslash A_{1}\right) B\right| \geq|A|+2|B|-2>|A|+|B|+k .
$$

Therefore we may assume that $B$ is not contained in a right coset of any cyclic subgroup.

If $|A| \leq k+3$, then let $A_{0}=A$, and if $|A|>k+3$, then let $A_{0}$ be a $(k+3)$-atom for $B$ with $1 \in A_{0}$. By definition, $|A B| \geq|A|+\left|A_{0} B\right|-\left|A_{0}\right|$. Proposition 9 gives that either $\left|A_{0} B\right|-\left|A_{0}\right|>|B|+k$, or $\left|A_{0}\right| \leq(k+3)(2 k+$ $3)$. If $\left\langle A_{0}\right\rangle$ is not cyclic, then choose $u, v \in A_{0} \backslash 1$ such that $\langle u, v\rangle$ is not cyclic. For $\widetilde{A}_{0}=\{1, u, v\} \subset A_{0}$, we have, by Lemma 11, that

$|A B| \geq|A|+\left|A_{0} B\right|-\left|A_{0}\right| \geq|A|+\left|\widetilde{A}_{0} B\right|-\left|A_{0}\right|>|A|+\left(|B|+2(k+3)^{2}\right)-\left|A_{0}\right|>|A|+|B|+k$.

Finally if $\left\langle A_{0}\right\rangle$ is cyclic, then $A_{0} \neq A$, so $\left|A_{0}\right| \geq k+3$, and $B$ intersects at least two right cosets of $\left\langle A_{0}\right\rangle$. Let $B_{1}$ be one of these intersections. We have by (4)

$|A B| \geq|A|+\left|A_{0} B\right|-\left|A_{0}\right|=|A|+\left|A_{0} B_{1}\right|+\left|A_{0}\left(B \backslash B_{1}\right)\right|-\left|A_{0}\right| \geq|A|+|B|+\left|A_{0}\right|-2>|A|+|B|+k$, completing the argument. Q.E.D.

If $G$ is a unique product group, then the argument above, just using Conjecture 10 in place of Proposition 9, leads to

Lemma 12 Let $G$ be a unique product group, $A, B \subset G$ finite subsets, and $k \geq 1$. Suppose that $A$ is not contained in a left coset of any cyclic subgroup, and $|B|>4(2 k+3)^{3}$, then

$$
|A B|>|A|+|B|+k .
$$


Acknowledgement: We are grateful for the help of Miklós Abért, Warren Dicks, Gábor Elek and Imre Ruzsa in the preparation of this manuscript. We particularly thank Yahya O. Hamidoune for fruitful discussions on the problem addressed in this paper, and Peter A. Linnell for providing in depth information on unique product groups.

\section{References}

[1] L.V. Brailovsky, G.A. Freiman: On a product of finite subsets in a torsion-free group. J. Algebra, 130 (1990), 462-476.

[2] E. Breuillard, B. Green: Approximate groups, I: the torsion-free nilpotent case. arXiv:0906.3598

[3] W. Carter: Non-unique product groups on two generators. Masters Thesis, Virginia Tech, 2003.

[4] T. Delzant: Sur l'anneau d'un groupe hyperbolique. C. R. Acad. Sci. Paris Sér. I Math., 324 (1997), 381-384.

[5] G.A. Freiman: Foundations of a structural theory of set addition. Translations of Mathematical Monographs, Vol 37. American Mathematical Society, Providence, R. I., 1973.

[6] G.A. Freiman: Structure theory of set addition. II. Results and problems. In: Paul Erdős and his mathematics, I, 243-260, Bolyai Soc. Math. Stud., 11, János Bolyai Math. Soc., Budapest, 2002.

[7] G.A. Freiman: Inverse additive number theory. XI. Long arithmetic progressions in sets with small sumsets. Acta Arith., 137 (2009), 325331.

[8] R.J. Gardner, P. Gronchi: A Brunn-Minkowski inequality for the integer lattice. Trans. Amer. Math. Soc., 353 (2001), 3995-4024.

[9] B. Green, T. Tao: Compressions, convex geometry and the FreimanBilu theorem. Quarterly J. Math., 57 (2006), 495-504.

[10] S.M. Hair: New methods for finding non-left-orderable and unique product groups. Masters Thesis, Virginia Tech, 2003.

[11] Y.O. Hamidoune: An isoperimetric method in additive theory. J. Algebra, 179 (1996), 622-630. 
[12] Y.O. Hamidoune: Some additive applications of the isoperimetric approach. Ann. Inst. Fourier (Grenoble), 58 (2008), 2007-2036.

[13] Y.O. Hamidoune: The isoperimetric method. In: Combinatorial number theory and additive group theory, Adv. Courses Math. CRM Barcelona, Birkhäuser Verlag, Basel, 2009, 241-252.

[14] Y.O. Hamidoune, A.S. Lladó, O. Serra: On subsets with small product in torsion-free groups. Combinatorica, 18 (1998), 529-540.

[15] J.H.B. Kemperman: On complexes in a semigroup. Nederl. Akad. Wetensch. Proc. Ser. A. 59, Indag. Math., 18 (1956), 247-254.

[16] M. Matolcsi, I.Z. Ruzsa: Sumsets and the convex hull. In: D. Chudnovsky, G. Chudnovsky (Eds.), Additive Number Theory, Festschrift In Honor of the Sixtieth Birthday of Melvyn B. Nathanson, Springer, accepted.

[17] S.D. Promislow: A simple example of a torsion-free, nonunique product group. Bull. London Math. Soc., 20 (1988), 302-304.

[18] E. Rips, Y. Segev: Torsion-free group without unique product property. J. Algebra, 108 (1987), 116-126.

[19] D.J.S. Robinson: A course in the theory of groups. 2nd ed., Springer, 1996.

[20] I.Z. Ruzsa: Sum of sets in several dimensions. Combinatorica, 14 (1994), 485-490.

[21] I.Z. Ruzsa: Sumsets and structure. In: Combinatorial number theory and additive group theory, Adv. Courses Math. CRM Barcelona, Birkhäuser Verlag, Basel, 2009, 87-210.

[22] A. Strojnowski: A note on u.p. groups. Comm. Algebra, 8 (1980), 231234.

[23] T. Tao: Non-commutative Freiman theorems, and model theory. (Blog) http://terrytao.wordpress.com/2009/12/06/

[24] T. Tao, V. Vu: Additive combinatorics, Cambridge University Press, 2006. 
Károly J. Böröczky, carlos@renyi.hu

Alfréd Rényi Institute of Mathematics, and

Universitat Politècnica de Catalunya, Barcelona Tech, and

Department of Geometry, Roland Eötvös University

Péter P. Pálfy, ppp@renyi.hu

Alfréd Rényi Institute of Mathematics

Oriol Serra,oserra@ma4.upc.edu

Universitat Politècnica de Catalunya, Barcelona Tech 